\title{
The Effectiveness of Constraints-Led Training on Skill Development in Football
}

\author{
Tiziana D'Isanto, Felice Di Domenico, Francesca D'Elia, Sara Aliberti*, Giovanni Esposito \\ Department of Political and Social Studies, University of Salerno, Salerno, 84084, Fisciano, Italy
}

Received August 30, 2021; Revised October 15, 2021; Accepted November 5, 2021

\begin{abstract}
Cite This Paper in the following Citation Styles
(a): [1] Tiziana D'Isanto, Felice Di Domenico, Francesca D'Elia, Sara Aliberti, Giovanni Esposito , "The Effectiveness of Constraints-Led Training on Skill Development in Football," International Journal of Human Movement and Sports Sciences, Vol. 9, No. 6, pp. 1344 - 1351, 2021. DOI: 10.13189/saj.2021.090630.
\end{abstract}

(b): Tiziana D'Isanto, Felice Di Domenico, Francesca D'Elia, Sara Aliberti, Giovanni Esposito (2021). The Effectiveness of Constraints-Led Training on Skill Development in Football. International Journal of Human Movement and Sports Sciences, 9(6), 1344 - 1351. DOI: 10.13189/saj.2021.090630.

Copyright $\bigcirc 2021$ by authors, all rights reserved. Authors agree that this article remains permanently open access under the terms of the Creative Commons Attribution License 4.0 International License

\begin{abstract}
This study aimed to examine whether constraints-led training improved technical skills in young football players. Twenty-four Under-13 amateur football players were divided into two groups: the experimental group (CLA; $\mathrm{n}=12$ ) participated in the Constraints-led training protocol; the control group $(\mathrm{n}=12)$ performed conventional training sessions based on the cognitive approach. The study consisted of a pre-test, two months of training intervention, and a post-test. Contextualized technical skills were tested using the Speed dribbling Test. The experimental CLA group recorded notable improvements in the observation variable, respectively: (d $[95 \% \mathrm{CI}]=0.90[0.70 ; 0.94] \mathrm{P}<0.05)$. The control group, on the other hand, did not show significant changes ( $\mathrm{P}>$ $0.05)$. From the ANOVA repeated measurements, it was possible to appreciate a significant group $\mathrm{x}$ moment interaction, respectively: $(\mathrm{d}[95 \% \mathrm{CI}]=0.77[0.54 ; 0.85]$ $\mathrm{P}<0.05)$. Participants who practiced with the constraint-driven training protocol demonstrated significant improvements in dribbling accuracy while reducing execution errors. This suggests that integrating CLA as a tool in a football training program can have a positive impact on their technical skills.
\end{abstract}

Keywords Team Sports, Game Intelligence, Motor Skills, Non-Linear Pedagogy

\section{Introduction}

Constraints-led Approach (CLA) looks quite promising for teaching sports technique, performance enhancement and talent development [2], however, the transition from pure theory to practice is not that simple. CLA was first mentioned in 1994 [6]. In over twenty years we have gone from the development of a theoretical framework to the attempt to apply the theory more in practice in relation to the target audience. Philosophical and theoretical clarity has been provided by the extensive literature in the area of non-linear pedagogy $[3,4,13]$. More recently, the focus is on sport in developmental age and in the high level. The beneficiaries of these studies are certainly the coaches and teachers who, in turn, can contribute by practicing CLA in their activities, reporting what comes from "knowledge".

CLA is based on the ecological-dynamic theories that consider athletes and sports teams as complex adaptive systems, in which the multitude of parts that constitute them, continuously form coordinated (or synergistic) models that are modified by surrounding information constraints [24]. The parts of a complex adaptive system can form synergies, as the individual parts that compose it can self-organize to achieve the required goal without the need for conscious control. The term constraint does not refer to the widespread meaning in the common use of limiting or negative factor, rather it should be understood as a border that shapes the structure of a biological system in search of a state of functional organization (i.e., of a state of organization that can help the subject to achieve the 
goals) [27].

One of the key concepts on which CLA is based is the relationship between perception and action [10], which underlies how constraints shape athletes' behaviors during sports practice and performance. Much emphasis is placed on the role of the environment or context; this means that the athlete-environment relationship forms the basis for understanding performance and development, as opposed to focusing attention only on personal qualities $[1,15]$. The way in which the environment presents itself to the athlete provides the latter with some opportunities that the subject must exploit in order to be successful.

In the theories of ecological dynamics, more than skill acquisition, we speak of skill adaptation, to describe the acquisition by the subject of an increasingly functional relationship with a particular performance environment [20]. The basis of dexterity according to Bernstein is to possess stable but flexible behavior patterns (understood not as a loss of stability, but as a sign of adaptation of skills), able to adapt to the changing needs of sports performance.

Among the disciplines in which there have been attempts to apply the CLA, football is also included [25]. The main problem is that young players very often participate in untrained competitions that create unfair habits, greatly compromising future performances. The technicians often resort only to prescriptive teaching methods, asking their athletes to perform stereotypical exercises, often without the intervention of the opponent. In this way, a relationship of maximum dependence is created between technician and student [22]. Analytical teaching should be limited in favor of expressiveness, balancing the developmental deficiencies that manifest themselves in learning. An improvement in perception and correct analysis of game situations for appropriate decision-making and, subsequently, effective technical execution of the intended action, are the essential prerequisites for improving the level of football in the future [21]. This study aims to verify whether through the use of Constraints-led training a group of young players can significantly improve the technical ability of dribbling compared to the traditional cognitive approach.

\section{Materials and Methods}

\subsection{Participants}

Twenty-four $(\mathrm{n}=24)$ young footballers (age: $12 \pm 1.2$; height; $170.2 \pm 0.5$; weight: $72 \pm 2.3$ ) belonging to the "esurient" category, were randomly assigned to an experimental group (which used the CLA) and to a control group (which used the cognitive approach). The experimental procedure, risks and benefits were explained to the athletes prior to participation. A consent form was signed by the athletes' parents and / or legal guardians.
This preliminary study adhered to the ethical code of the Declaration of Helsinki and the procedures were in line with the ethical standards established in the sports sciences (Winter \& Maughan, 2009).

\subsection{Study Design}

A longitudinal design was employed to test the difference in technical skills between the two groups over time. A pre-test was carried out in October while the post-test was carried out after two months of differentiated training (in December). The experimental group carried out a program of structured exercises based on the CLA. The control group performed a conventional training program prescribed by the coach.

\subsection{CLA Training Sessions}

To implement training that complies with the principles of the CLA, three categories of constraints must be considered, which will then be modified according to the specific needs of the session:

- Individual constraints: they concern the personal characteristics of each individual such as genes, weight, height, motivations, amount of experience, desires, etc.

- Environmental Constraints: these can relate to physical variables such as ambient light, temperature, altitude, field size, or social characteristics such as values and beliefs.

- Task constraints: these relate to game/training objectives, type of exercise, specific rules associated with an activity, use of tools or tools related to the activity, etc.

By manipulating these constraints, learning experiences can be designed that are valid and that guarantee optimal transfer in the competition environment. The CLA was implemented during each training session, 2 sessions per week for 2 months, and adequate space was dedicated to it, about $1 / 3$ of the overall training session. Different types of training sessions have been implemented, considering the starting level of their players. Sessions focused on:

- Ball possession: The exercise, shown in Figure 1, provides that the players, each with their own ball, ran at a medium-high intensity avoiding their teammates. Already here there was a manipulation of constraints such as: the specific type of exercise, the objective to be pursued, the specific rules. Additional constraints could be added such as: placing cones on the ground inviting players to avoid them, or reducing the field, thus also acting on environmental constraints and stimulating greater opportunities for contact between the players. 


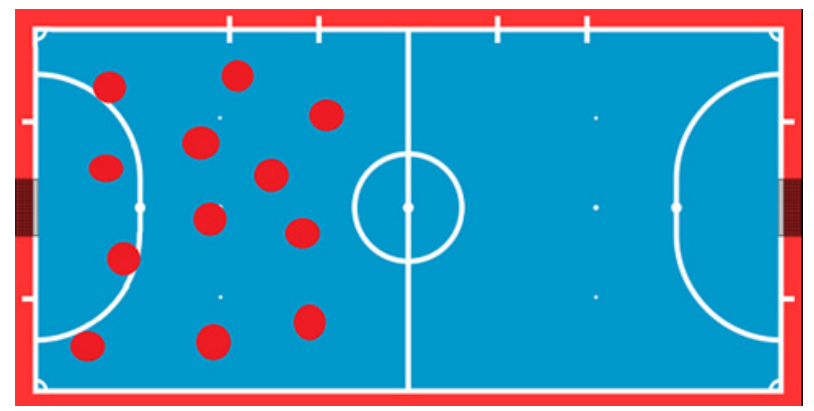

Figure 1. Ball possession exercises

- $\quad$ Small Sided Games: as Figure 2 shows, the real 2v2 and $3 \mathrm{v} 3$ minigames were played in small spaces with cones delimiting the net. The constraints manipulated in this activity concern: the number of players involved, space, time, the type of exercise and the goal of the game.

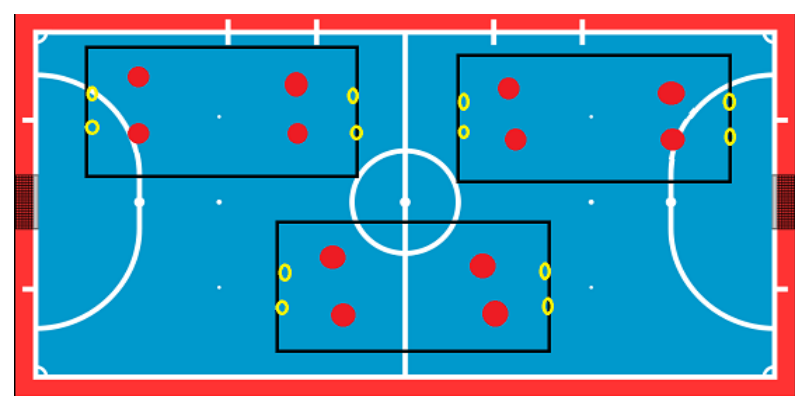

Figure 2. Small-Sided Games

- Trigger-Runner: activity in which the player in possession of the ball tries to escape from the pursuer in a confined space trying to preserve possession of the ball. The pursuer is also in possession of the ball. The constraints manipulated in this activity concerned: the number of players involved, space, time, the type of exercise, and the goal and rules of the game. You can further modify the activity by removing the ball from the pursuer, or by adding ball carrier, that is another player. The scheme of the exercise is represented in Figure 3.

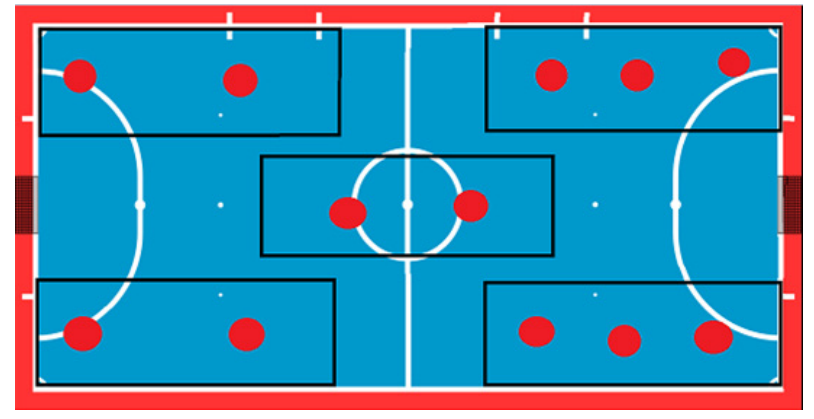

Figure 3. Trigger-runner exercise
All these sessions, although different from each other, had the aim of placing the players in situations where dribbling was necessary, even without imposing it as an explicit or sole objective of the exercise.

\subsection{Traditional Training Sessions}

The control group took part in a training program based on the cognitive approach. The method of exercise by blocks was used, the one most frequently used in football. The exercise by blocks consists of a sequence of activities in which the same task is always repeated. They are sequential, timed exercises, in which the goal is clearly explained by the teacher and does not change. The belief is that it is useful to focus one's attention and efforts only on one task and then move on to another one only when the first has been analyzed, corrected, perfected and assimilated. The block exercises were performed by the control sample twice a week for 2 months. Exercises have been structured such as:

- Dribbling around the cones: exercise in which the players had to perform the technical gesture at maximum speed in a dedicated circuit. The coach gave the starting signal to each player and between one repetition and the next there were 2 minutes of active recovery. The exercise included 3 series of 3 repetitions. The scheme of the exercise is represented in Figure 4.

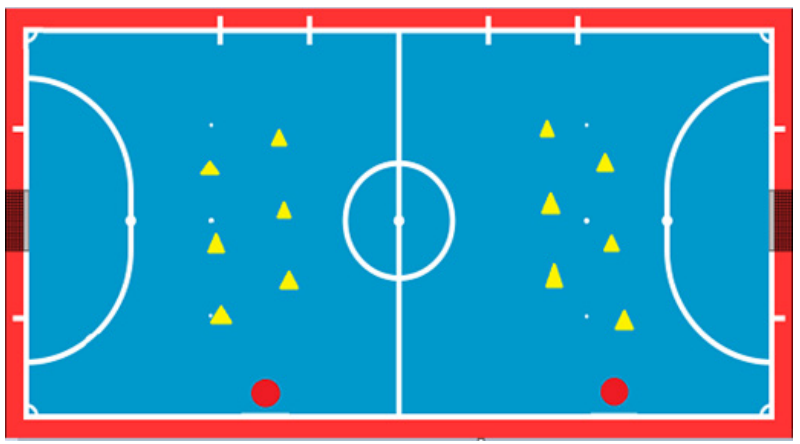

Figure 4. Dribbling exercise 1

- Dribbling with course: the players were divided into 4 micro groups. The coach gave the starting signal. The exercise is shown in Figure 5. It provided that two players, in a direction not parallel to each other, began to guide the ball from their initial position, delimited by some cones, proceeding parallel to their own trajectory. Once they got close to the cones, both players had to dribble them, being careful not to collide with each other. After passing the cones they passed the ball to the partner placed in front, positioning themselves behind him. The exercise lasted 3 minutes and included 2 series of 3 repetitions. 


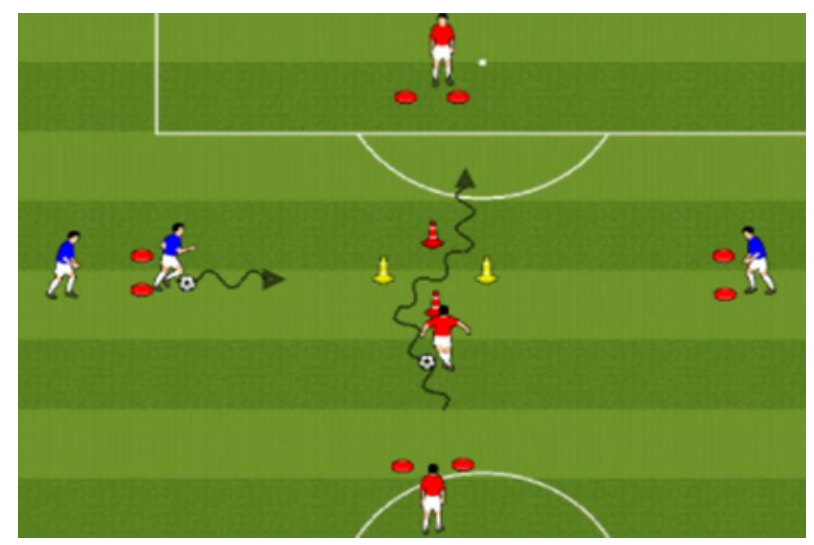

Figure 5. Dribbling exercise 2

- Soccer Dribble-Pass: it is an exercise that is performed on one of the two half-fields. As can be seen in Figure 6, the players are positioned equally in the 4 vertices, each with a ball. Each player had to carry the ball clockwise towards his teammate by dribbling a cone placed in the middle of the course and then passing the ball to the teammate placed in front. The exercise lasted 5 minutes and included 2 sets of 5 repetitions.

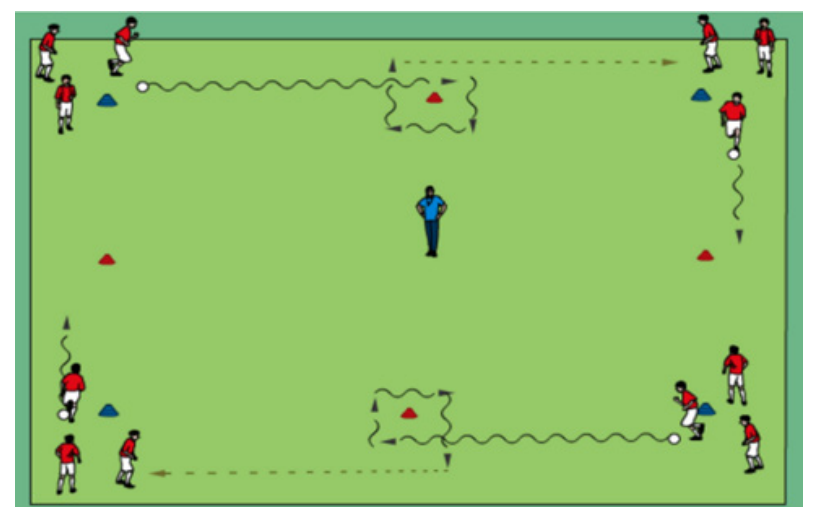

Figure 6. Soccer Dribble-pass

\subsection{Field Test}

A field test was performed before the training period, and two months after the training period to assess the dribbling ability of the players. The test selected is the
Speed dribbling test, belonging to the F-MARC battery [18]. It allows the evaluation of dribbling time and speed. The test scheme is shown in Figure 7.

After the start signal, the players began to lead the ball forward for $5 \mathrm{~m}$, after which he performed a series of dribbles (changes of direction at various angles) around 6 flags (or cones) arranged in a triangle in a space of $5 \mathrm{~m}$. Then he continued for another $10 \mathrm{~m}$ until he reaches a cube around which he had to turn, still in possession of the ball, to arrive after another $8 \mathrm{~m}$ to another cube of about $2 \mathrm{~m}$ on each side. Here the ball had to be passed on one side while the player dribbled the obstacle on the other side; then resumed the sphere headed towards a door located at $20 \mathrm{~m}$. The test ends when he stopped the ball after passing the goal. Three attempts were made, taking as a reference the best time taken by the player to complete the course. Table 1 shows the reference values of the test found in the literature.

Table 1. Test reference values found in the literature (in relation to age $10-12)$

\begin{tabular}{|c|c|c|c|}
\hline Poor & Average & Good & Excellent \\
\hline$>50 \mathrm{sec}$ & $40<\mathrm{x}<50 \mathrm{sec}$ & $30<\mathrm{x}<39 \mathrm{sec}$ & $<30 \mathrm{sec}$ \\
\hline
\end{tabular}

\subsection{Statistical Analysis}

Data were reported as mean and standard deviation. Through the Shapiro Wilk test it was initially ascertained that the data had a normal distribution within each group and time of assessment $(\mathrm{P}>0.05)$. A first t-test for independent samples was performed to assume a non-significant difference between the values recorded by the groups before carrying out the training program. A further independent sample t-test was performed to evaluate the differences between the groups after two months of training. A repeated measures analysis of variance (ANOVA) was used to test for training-induced changes in the observation variable. To qualitatively interpret the magnitude of the differences, the effect size (d) and the associated $95 \%$ confidence intervals $(95 \% \mathrm{CI})$ were classified as small $(0.2-0.5)$, moderate $(0.5-0.8)$ and large $(>0.8)$. Data analyses were performed using Statistical Package for Social Science software (IBM SPSS Statistics for Windows, version 27.0. Armonk, NY).

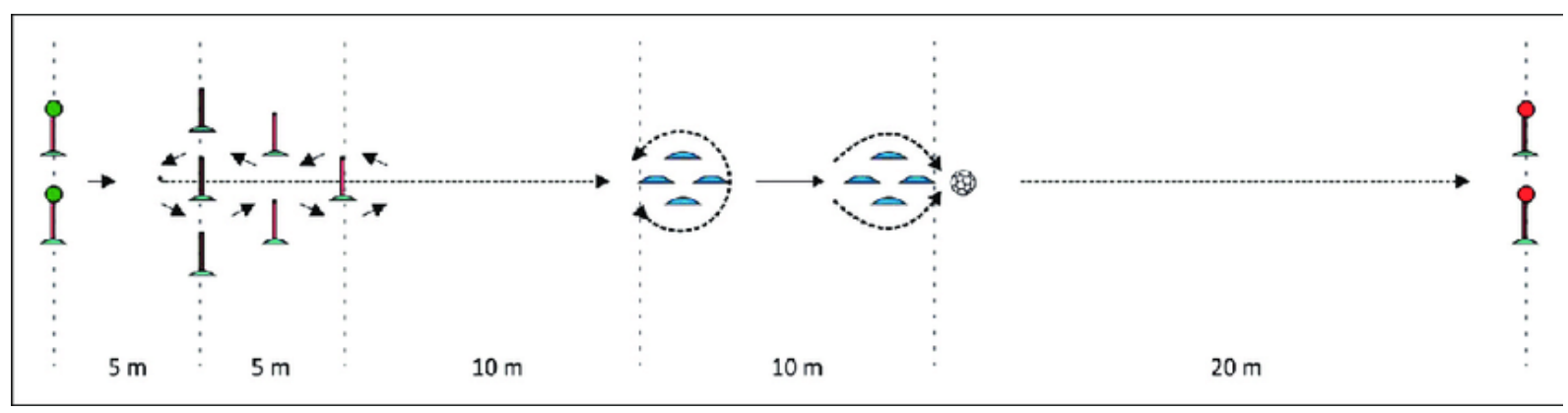

Figure 7. Speed dribbling test 
Table 2. Data recorded by the players in the speed dribbling test

\begin{tabular}{|c|c|c|c|c|}
\hline & \multicolumn{2}{|c|}{ CLA group } & \multicolumn{2}{c|}{ Control group } \\
\hline Players & $\begin{array}{c}\text { Before training period } \\
(\mathbf{s})\end{array}$ & $\begin{array}{c}\text { After training period (s) } \\
\text { Player 1 }\end{array}$ & $\begin{array}{c}\text { Before training period } \\
\text { (s) }\end{array}$ & After training period (s) \\
\hline Player 2 & 31.25 & 35.40 & 37.65 & 36.65 \\
\hline Player 3 & 47.47 & 32.30 & 48.50 & 47.30 \\
\hline Player 4 & 37.35 & 36.26 & 41.60 & 39.88 \\
\hline Player 5 & 44.26 & 31.20 & 35.21 & 33.90 \\
\hline Player 6 & 34.64 & 40.11 & 49.05 & 47.41 \\
\hline Player 7 & 40.42 & 28.10 & 33.10 & 35.86 \\
\hline Player 8 & 39.34 & 33.10 & 38.20 & 38.10 \\
\hline Player 9 & 33.21 & 32.47 & 38.70 & 38.50 \\
\hline Player 10 & 38.85 & 27.72 & 35.47 & 36.10 \\
\hline Player 11 & 42.52 & 33.67 & 41.90 & 41.07 \\
\hline Player 12 & 53.52 & 31.82 & 34.22 & 34.03 \\
\hline Mean + SD & $40.9 \pm 5.5$ & 42.02 & 45.52 & 45.29 \\
\hline
\end{tabular}

\section{Results}

All players took the test both before and after the training period. A detailed description of the individual scores in the speed dribbling test is given in Table 2.

After having ascertained the normal distribution of the data through the Shapiro-Wilk test, it was possible to perform the parametric test of the t-test for independent samples to compare the means of the two groups before and after training. The test results are shown in Table 3 and 4.

Table 3. T-test for independent samples to assume insignificant differences between values before the training program

\begin{tabular}{|c|c|c|c|c|c|c|}
\hline Group & N & Mean & SD & SE & t & p \\
\hline CLA & 12 & 40.96 & 5.54 & 1.60 & .464 & .648 \\
\hline CON & 12 & 39.92 & 5.44 & 1.57 & & \\
\hline
\end{tabular}

There was no statistically significant difference between the two groups before the training period, $p>$ 0.05 .

Table 4. Independent sample T-tests to evaluate the differences between groups in the post-intervention period

\begin{tabular}{|c|c|c|c|c|c|c|}
\hline Group & $\mathbf{N}$ & Mean & SD & SE & $\mathbf{t}$ & $\mathbf{p}$ \\
\hline CLA & 12 & 33.68 & 4.26 & 1.23 & -3.13 & .005 \\
\hline CON & 12 & 39.50 & 4.83 & 1.39 & & \\
\hline
\end{tabular}

A statistically significant difference was instead recorded by comparing the results obtained by the two groups at the end of the training period, $\mathrm{p} \leq 0.05$.

To examine the influence of the training intervention on the development of our dependent variable (the score obtained from the test), repeated measures ANOVA was used to test the veracity of the null hypothesis that predicted a change over time between groups (moment $\mathrm{x}$ group interaction). The design used is the " $2 \times 2$ " model, where we had two factors each of which could assume two different levels. The two independent variables included a factor between the subjects (the different training protocol performed by the experimental and the control group), and a factor within the subjects: the time with the two levels of development (pre- and post-intervention). A detailed description of the improvements recorded after the training period has been reported in Table 5.

It is possible to observe how the CLA experimental group recorded notable improvements in the observation variable, respectively: $(\mathrm{d}[95 \% \mathrm{CI}]=0.90[0.70 ; 0.94] \mathrm{P}$ $<0.05$ ). On the other hand, the control group showed no significant changes $(\mathrm{P}>0.05)$. From the repeated measures ANOVA, it was possible to appreciate a significant group $\mathrm{x}$ moment interaction, respectively: $(\mathrm{d}[95 \% \mathrm{CI}]=0.77$ $[0.54 ; 0.85] \mathrm{P}<0.05)$. 
Table 5. Comparison of technical performance after football training with the CLA approach and with the cognitive approach (CON)

\begin{tabular}{|l|c|c|c|c|c|c|}
\hline & \multicolumn{2}{|c|}{ CLA $(\boldsymbol{n}=\mathbf{1 2})$} & \multicolumn{2}{c|}{ CON $(\boldsymbol{n}=12)$} & \multicolumn{2}{c|}{ Group x Moment interaction } \\
\hline Variable & $p$ & $\mathrm{~d}(95 \% \mathrm{CIs})$ & $p$ & $\mathrm{~d}(95 \% \mathrm{CIs})$ & $p$ & $\mathrm{~d}(95 \%$ CIs $)$ \\
\hline Trial (s) & 0.001 & $0.90(0.70 ; 0.94)$ & 0.261 & $0.11(0.01 ; 0.43)$ & 0.001 & $0.77(0.54 ; 0.85)$ \\
\hline
\end{tabular}

\section{Discussion}

The purpose of this preliminary study was to evaluate whether a training program based on the CLA approach could have a greater impact on the improvement of the technical skills of young players than the traditional use of the cognitive approach. The results obtained by the experimental group suggest that the introduction and manipulation of some constraints in the design of the training session are able to further encourage athletes to explore opportunities for action in relation to the intention of the session, resulting in positive effects on performance. On the other hand, no significant changes were observed for the control group over time. The concept of a representative learning environment questions the value of those practical activities that are decontextualized through artificiality and reductionism in a performance environment. To exemplify, in practical learning interventions, it is important not to design an environment that requires learners to dribble around cones or manikins to create realism - as this lacks the subtle informational constraints that authenticate valid practices, thus rendering a practice as lacking representativeness. Without the information of opposing defenders, spatial (line markings) or temporal (time of a ball feed) informational constraints there will be the little strengthening of the perception-action couplings required in skilled performance.

The activities proposed with the use of the CLA have left free space for the creativity of the players without addressing them specifically towards certain behaviors. This is even more important when the objective is to be achieved, in this case dribbling is a fundamental technical that requires a great ability to self-organize (because it is carried out in a very short time) within a context that constantly varies (variability contextual) [11]. Furthermore, being able to train with or against one's teammates has allowed to focus the perception-action systems on important information sources that are also found in the competition context, thus also allowing an optimal transfer. Finally, the motivational aspect was also able to benefit, with players learning. They are happy to do it because they have fun (heuristic learning).

On the other hand, the activities proposed to the control group, carried out under the directives of the coach, without the execution of variations, allowed the boys to focus attention only on a limited number of elements, consequently they had a limited number of ways to do the exercise. This allowed only momentary learning of the skills, which did not prove to be lasting in which we went to carry out the post-training tests. Previous studies that used the constraint-led approach in player skill development have changed the constraints of the motor task to a greater extent [14] such as the variation of the execution technique [7], scaling the equipment, the size of the field and the height of the net [16]. Some studies have focused on the manipulation of a single aspect within the task to provide individuals with alternative means of learning task-relevant skills. For example, Stretch et al., [26] attempted to train cricket batsmen to improve their accuracy of hitting the ball in the middle of the bat; this was done through the use of a modified bat, one-third the width of the regulation size. However, CLA may make use of multiple constraints manipulations in order to provide individuals with improved training methods while maintaining the principles of the ecological dynamics approach. Therefore, the practical tasks that are meant to prepare players for game performance should simulate space-time variables true to the dynamics of the game. Previous studies have suggested that intrinsically timed closed exercises that produce highly repeatable actions risk diminishing the ability of footballers to develop flexible and adaptable movement solutions $[12,19]$. Farrow et al., [8] compared the physiological skills and needs of open and closed exercises commonly used in Australian football and found that there was a significantly greater volume of game decisions, and consequently the cognitive training load, in the specific game-context of open exercises. These results suggest the need for a pedagogical approach in which the coach can be creative in the design of training games, play practices, and match simulations [5]. This, in turn, would allow for information-movement coupling leading to the emergence of functional motion solutions to the problems presented in momentary game configurations [17]. In other words, the coach's challenge is to design practice environments that provide controlled boundaries of exploration in dynamic contexts through the provision of relevant constraints [23]. The development of the game's abilities and dimensions depends significantly on the approach to teaching coaches [28].

It is important to define some limitations of this study. First of all, it is necessary to highlight the small sample size and the reduced training period carried out. Secondly, a retention test could not be performed a few weeks after the second test was performed. Through the retention test, we could verify if the significant improvements observed were maintained over time as a learning effect. Another important limitation is the variability of biological maturation in the under-13 [9]. Inter-individual variations in growth, maturation, functional capacity, and specific 
skills in youth football must be considered. This could justify the extreme heterogeneity of the results in the control group. Further research should consider implementing better quality control in methodological design. For small samples, the use of cross-test designs should be considered to enhance the reliability of the results. Retention tests could be used following surgery. Depending on the type of intervention, the participants' skills and/or experience level are important factors to consider.

\section{Conclusions}

CLA proved to be more effective than the cognitive approach to improving the dribbling ability of children. This preliminary study should lead the coaches to consider a variation/change of methodological approach, based on the principles of CLA, allowing to strengthen the perception-action couplings, self-emergent behaviors, develop creativity, allow an effective transfer of skills in the competition context and entertain kids by manipulating constraints.

\section{REFERENCES}

[1] Brymer E., Renshaw I., "An introduction to the constraints-led approach to learning in outdoor education". Journal of Outdoor and Environmental Education, vol. 14, no. 2, pp. 33-41, 2010, DOI: 10.1007/BF03400903

[2] Chow J.Y., Davids K., Button C., Shuttleworth R., Renshaw I., Araujo D., "The role of nonlinear pedagogy in physical education". Review of Educational Research, vol. 77, no. 3, pp. 251-278, 2007, DOI: 10.3102/003465430305615

[3] Chow J.I., Davids K., Button C., Renshaw I., "Nonlinear Pedagogy in Skill Acquisition: An Introduction", Routledge, London, 2016.

[4] Davids K., Button C., Bennett S., "Dynamics of Skill Acquisition: A constraints-led approach", Human Kinetics, Champaign IL, 2008.

[5] Davids K., Araújo D., Shuttleworth R., "Applications of dynamical systems theory to football", in Science and football V, Routledge, pp. 570-572, 2005.

[6] Davids K., "The constraints-based approach to motor learning: Implications for a non-linear pedagogy in sport and physical education", in Motor learning in practice, Routledge, pp. 23-36, 2010.

[7] Farrow D., Pyne D., Gabbett T., "Skill and physiological demands of open and closed training drills in Australian football", International Journal of Sports Science and Coaching, vol. 3, no. 4, pp. 489-499, 2008, DOI: $10.1260 / 174795408787186512$

[8] Farrow D., Reid M., "The effect of equipment scaling on the skill acquisition of beginning tennis players", Journal of sports sciences, vol. 28, no. 7, pp. 723-732, 2010.

[9] Figueiredo A.J., Gonçalves C.E., Coelho e Silva M.J., Malina R.M., "Characteristics of youth soccer players who drop out, persist or move up", Journal of sports sciences, vol. 27, no. 9, pp. 883-891, 2009, DOI: $10.1080 / 02640410902946469$

[10] Gibson J.J., "James J. Gibson”, in A History of Psychology in Autobiography, vol. 5, Appleton-Century- Crofts, pp. 127-143, 1967.

[11] Giordano L., Federici A., Valentini M., D'Elia F., "Dribbling in football: Confronting learning theories", Journal of Human Sport and Exercise, vol. 14, no. 2, pp. S228-S232, 2019, DOI: $10.14198 /$ jhse.2019.14.Proc2.10

[12] Gorman A., "Using constraints to enhance decision-making in team sports" in Motor learning in practice: A constraints-led approach, Routledge, pp. 144-151, 2010.

[13] Handford C.H., Davids K., Bennett S., Button C., "Skill acquisition in sport: Some applications of an evolving practice ecology", Journal of Sport Science, vol. 19, no. 4, pp. 321-349, 1997.

[14] Hernández-Davo H., Urbán T., Sarabia J.M., Juan-Recio C., Javier Moreno F., "Variable training: effects on velocity and accuracy in the tennis serve", Journal of sports sciences, vol. 32, no. 14, pp. 1383-1388, 2014, DOI: $10.1080 / 02640414.2014 .891290$.

[15] Invernizzi P.L., Crotti M., Bosio A., Cavaggioni L., Alberti G., Scurati R., "Multi-teaching styles approach and active reflection: Effectiveness in improving fitness level, motor competence, enjoyment, amount of physical activity, and effects on the perception of physical education lessons in primary school children", Sustainability, vol. 11, no. 2, 2019.

[16] Kachel K., Buszard T., Reid M., "The effect of ball compression on the match-play characteristics of elite junior tennis players", Journal of sports sciences, vol. 33, no. 3, pp. 320-326, 2015.

[17] Komar J., Potdevin F., Chollet D., Seifert L., "Between exploitation and exploration of motor behaviors: Unpacking the constraints-led approach to foster nonlinear learning in physical education", Physical Education and Sport Pedagogy, vol. 24, no. 2, pp. 133-145, 2019, DOI: $10.1080 / 17408989.2018 .1557133$

[18] Padron-Cabo A., Rey E., Pérez-Ferreirós A., Kalén A., "Test-Retest Reliability of Skill Tests in the F-MARC Battery for Youth Soccer Players", Perceptual and motor skills, vol. 126, no. 5, pp. 1006-1023, 2019.

[19] Raiola G., Lipoma M., Tafuri D., "Postural control in young soccer players: differences between the cognitive approach and ecological-dynamic one" Journal of Human Sport and Exercise, vol. 10, no. 1, pp. S385-S390, 2015, DOI: 10.14198/jhse.2015.10.Proc1.29

[20] Raiola G., "Motor control and learning skills according to cognitive and ecological dynamic approach in a vision on behaviorism, cognitive, Gestalt and phenomenology theories", Mediterranean Journal of Social Sciences, vol. 5, no. 15 , pp. 504-504, 2014.

[21] Ramos A., Coutinho P., Davids K., Mesquita I., "Developing Players' Tactical Knowledge Using Combined 
Constraints-Led and Step-Game Approaches-A Longitudinal Action-Research Study", Research Quarterly for Exercise and Sport, pp.1-15, 2020, DOI: $10.1080 / 02701367.2020 .1755007$

[22] Renshaw I., Davids K.W., Shuttleworth R., Chow J.Y., "Insights from ecological psychology and dynamical systems theory can underpin a philosophy of coaching", International Journal of Sport Psychology, vol. 40, no. 4, pp. 540-602, 2009.

[23] Renshaw I., Chow J.Y., Davids K., Hammond J., "A constraints-led perspective to understanding skill acquisition and gameplay: A basis for the integration of motor learning theory and physical education praxis?", Physical Education and Sport Pedagogy, vol. 15, no. 2, pp. 117-137, 2010, DOI: 10.1080/17408980902791586.

[24] Renshaw I., Davids K., Newcombe D., Roberts W., "The constraints-led approach: Principles for sports coaching and practice design" Routledge, 2019.
[25] Sannicandro I., "Ecological dynamics approach in the youth soccer: A short narrative review", Journal of Human Sport and Exercise, vol. 15, no. 4, pp. S1133-S1139, 2020.

[26] Stretch R.A., Nurick G.N., McKellar D., "Improving the accuracy and consistency of shot reproduction in cricket batting”, South African Journal for Research in Sport, Physical Education, and Recreation, vol. 21, no. 1, pp. 77-88, 1998, DOI: 10.1080/17461391.2015.1094145.

[27] Uehara L., Button C., Davids K., "Sport expertise development and the constraints-led approach: a review exemplified by the case of Brazilian soccer", Conexões, vol. 17, no. 1, pp. 1-20, 2019.

[28] Vélez-Meza Edda, Méndez-Urresta Jacinto, Vargas-Chagna Johanna, Flores-Torres Diego, "Integral Analysis of Cognitive Motor Skills and the Social Dimension of Sport: Approach to Sports Initiation and School Sports," International Journal of Human Movement and Sports Sciences, Vol. 9, No. 3, pp. 495 - 502, 2021. DOI: 10.13189/saj.2021.090315. 